\title{
Spontaneous coronary artery dissection in an antilles man with acute inferior myocardial infarction
}

\author{
Gilbert Pierre-Justin $^{\mathrm{a}, *}$, Luc A. Pierard ${ }^{\mathrm{b}}$ \\ ${ }^{\text {a }}$ Department of Cardiology University Hospital Fort-de-France, France \\ ${ }^{\mathrm{b}}$ Department of Cardiology University Hospital Sart Tillman, Liege, Belgium \\ Received 30 May 2004; accepted 18 January 2005 \\ Available online 8 February 2007
}

\begin{abstract}
Spontaneous coronary artery dissection is a rare cause of myocardial infarction. It has been reported mainly in young women during or after pregnancy. The diagnosis is usually confirmed at autopsy. The management of these patients remains controversial. We report the case of a patient who presented spontaneous dissection and was with successfully treated by thrombolysis. Clinical outcome was good.

(C) 2006 Elsevier Ireland Ltd. All rights reserved.
\end{abstract}

Keywords: Coronary dissection; Myocardial infarction; Thrombolysis

Spontaneous coronary artery dissection is a rare cause of myocardial infarction, occurring as complication aortic dissection or occurring after blunt, chest trauma, coronary bypass grafting, cardiac catheterisation or coronary angioplasty. The diagnosis is frequently obtained at autopsy. The management is not yet well codified. We report the case of a patient who presented spontaneous dissection, with a good outcome after thrombolysis.

\section{Case report}

55-year old Antilles man, without previous cardiac history, was admitted in the intensive care unit for acute inferior myocardial infarction. Physical examination revealed blood pressure of $150 / 90 \mathrm{mmHg}$, pulse rate of $64 / \mathrm{min}$, a respiratory rate of $18 / \mathrm{min}$ and a normal cardiovascular examination serial electrocardiograms revealed inferior Q wave myocardial infarction, confirmed by characteristic increase and decrease of creatine-Kinase with peak serum level of 1474 IU/l. Chest X-ray was normal. He

\footnotetext{
* Corresponding author. Tel.: +33 675487598; fax: +33 0241867027.

E-mail address: gpierrejustin@Hotmail.com (G. Pierre-Justin).
}

received intravenous tissue plasminogen activator $5 \mathrm{~h}$ after the onset of pain followed by resolution of chest pain rapid resolution and of ST segment elevation (Figs. 1 and 2). Twodimensional echocardiography revealed hypokinesia of the inferior wall. A first catheterisation was performed 12 days later. Hypokinesia of left ventricular inferior wall was found at angiography. Coronary arteriography demonstrated a floating intimal flap in the right coronary artery indicating coronary artery dissection. No atherosclerotic plaque was observed. (Fig. 3). The collagen screen was negative for auto-immune disorders. Doppler of the carotid arteries and thoracoabdominal CT scan were normal. Twelve days later, the patient was discharged from the hospital on medical therapy.

\subsection{Follow-up}

Angiographic follow-up was performed 5 months later; there was no residual sign (Fig. 4). On a subsequent treadmill test, he achieved stage 4 of a standard Bruce protocol without symptoms or inducible ischemia. Dobutamine stress echocardiography and MIBI single photon emission tomography were normal. The patient had no symptoms throughout 18month follow-up. 


\section{Discussion}

Spontaneous dissection is a rare whose precise incidence, etiology, pathogenesis, and treatment have not been clearly established. It has been described as a possible cause of acute myocardial infarction and sudden death among young patients, especially women during the postpartum period.

Spontaneous coronary artery dissection has been reported in 183 patients in the worldwide literature the majority of patients were women $120(75 \%)$ and $63(35 \%)$ were men. The true incidence of spontaneous coronary artery dissection is most probably underestimated; this event may be followed by sudden death. Mean age was $41 \pm 11$ years at the time of the diagnosis (ranging from 17 to 71 years). There was no racial preponderance. Dissection appears to occur more frequently in the left anterior descending artery $(78 \%)$, in women, and the right coronary artery in the men (41\%). Multi-vessel involvement has been reported predominantly in women.

Spontaneous coronary artery dissection can be clinically silent or induces angina. Its intensity appears to be dependent on the location and extent of the dissection, and the subsequent severity of myocardial ischaemia.

The etiology of spontaneous coronary artery dissection remains unclear. DeMaio et al. [1] has reviewed the literature and collected a total of 83 cases including 62 antemortem and 21 postmortem diagnoses. Three groups of cases are distinguished: the first group consists in women in the postpartum period. It was suggested that the association of spontaneous coronary dissection with pregnancy and the postpartum period is due to an excess of progesterone during pregnancy which induces several structural abnormalities in coronary vessels walls, loss of normal corrugation in elastic fibers, fragmentation of reticular fibers, and marked transformation of muscular arteries. Koul et al. [2] suggested an additional role for the hemodynamic stress related to pregnancy, labor, and delivery causing coronary artery dissection in the peripartum period. These changes would be expected to return to normal by 3 months following delivery. The second etiology is atherosclerotic coronary disease: the dissection result from plaque rupture resulting in disruption of the junction between the intima and media. And an idiopathic group without an identifiable predisposing factor. Robinowitz et al. [3] have found in a postmortem study that their 8 patients aged 26 to 47 years and $31(43 \%)$ of the previously reported autopsy cases had an eosinophilic infiltrates in the adventitia of the involved artery. They postulated that proteins isolated from the granules of eosinophiles secreting lytic enzymes and major basic protein can damage collagen, elastin and smooth muscle cells in the coronary artery, resulting in dissection. Others, however, suggest that the inflammation is a consequence of the dissection and not the cause $[2,4]$. Other autopsy series have described that cystic spaces in the vessel media could potentially be the cause of dissection. Bonnet at al. [5] suggest that low collagen synthesis as in patients with Elhers-Danlos syndrome type IV or III might be responsible for an alteration in the coronary vessel walls, resulting in spontaneous coronary dissection these diseases are known to be due to an inborn defect in collagen metabolism. On the other hand, since an intimal tear was found only infrequently at autopsy, disruption of vasa vasorum leading to intramedial hemorrhage and subsequent dissection without intimal tear has been proposed as a possible mechanism. It has been suggested that a partial tear originating in the intima or adventitia may lead to medial damage, hemorrhage, and dissection. Disruption of intima or adventitia, however, is not mandating for traumatic formation of a hematoma in the media.

The prognosis of these patients has not been defined. In the follow-up of 27 patients in DeMaio's review reported in 1989 [1] :

- 2 patients $(7 \%)$ died immediately

- 3 others (11\%) died during follow-up at 48, 72, 132 months

- $22(82 \%)$, all these patients, except one remained symptom free.

Currently, Koul et al. [2] in this review of the world literature reported an overall mortality of $38 \%(22 / 58)$ in all

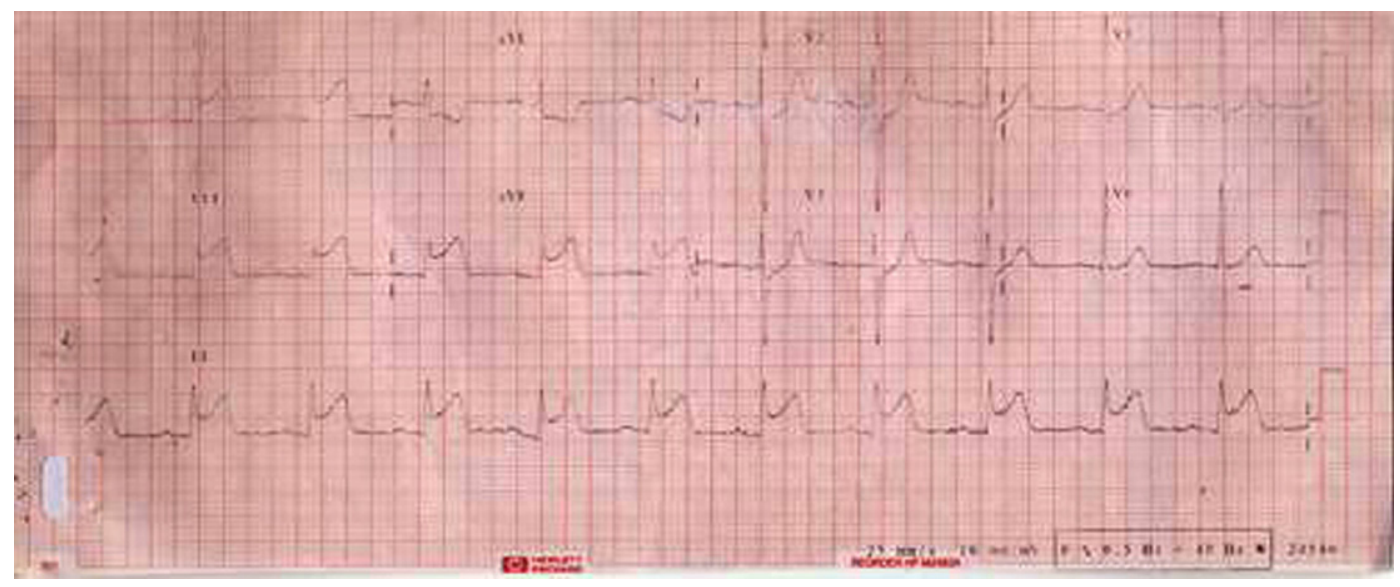

Fig. 1. ECG In the intensive care unit: appearances of acute inferior myocardial infarction. 


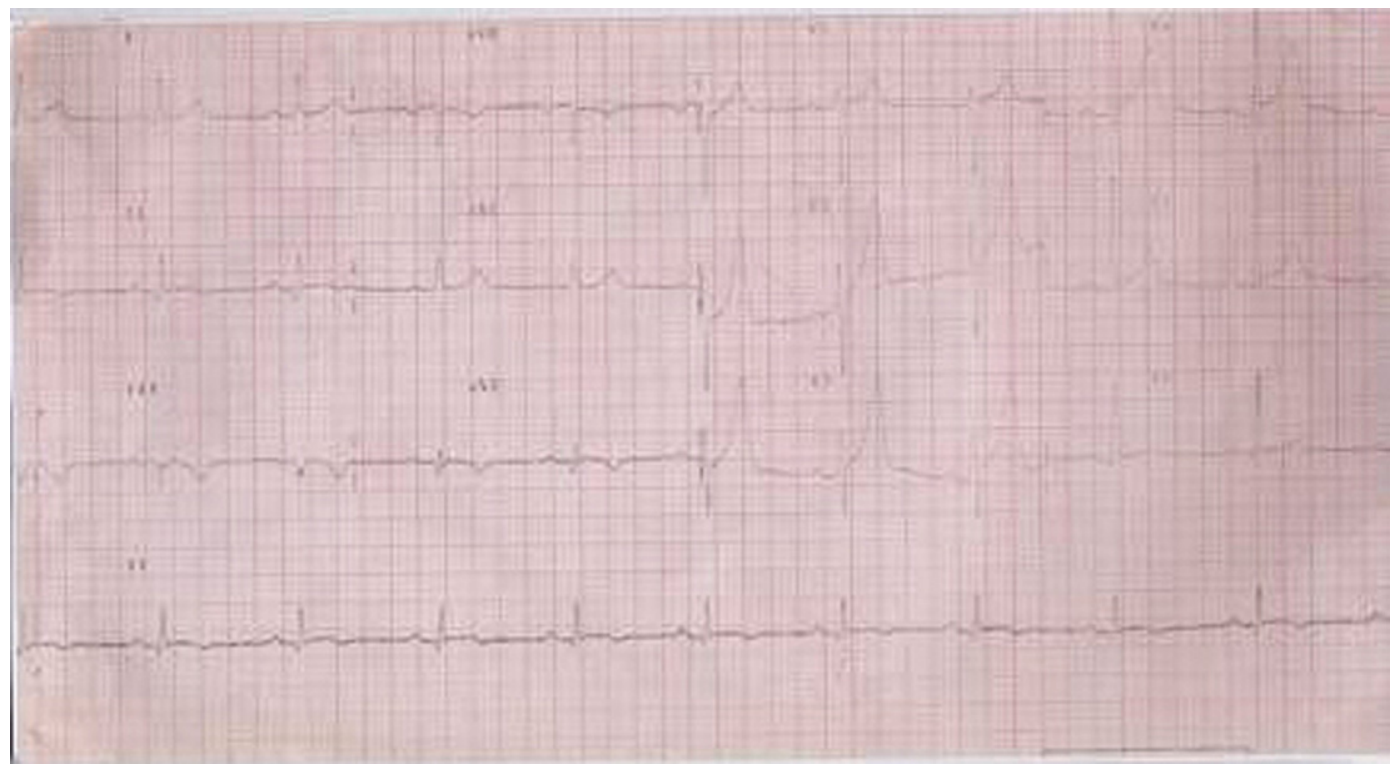

Fig. 2. ECG: rapid resolution of ST segment elevation after perfusion of intravenous tissue plasminogen activator.

reported cases. Half of the patients, who survived the initial event, were treated medical by therapy, and $30 \%$, underwent coronary artery bypass grafting. One patient had surgical extrusion of the hematoma from the LAD, $8 \%$ required cardiac transplantation, and $6 \%$ patients were treated with angioplasty and/or stenting. There was $0 \%$ mortality in both the medically and surgically treated groups. And he suggest that, the successful outcome in this clinical entity is encouraging and underscores the importance of an aggressive approach to diagnosis and treatment, particularly in young without risk factors.

The decision to treat spontaneous coronary artery dissection medically or surgically has not been previously addressed. Initial management of the patients has included intravenous heparin, nitro-glycerine, and medication for the relief of pain. Thrombolytics would appear to be relatively contraindicated in these patients as they may actually increase the risk of hemorrhage and further progression of the

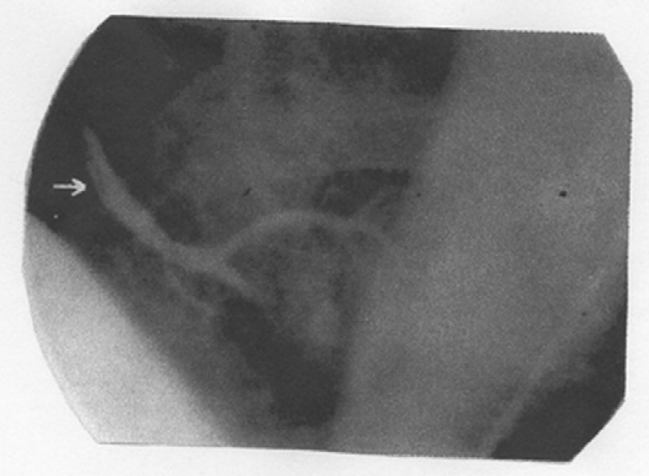

Fig. 3. Coronary arteriography demonstrated a floating intimal flap in the right coronary artery indicating coronary artery dissection. No atherosclerotic plaque was observed. dissection. Thrombolysis has been given in a few cases with favorable outcome including our patient $[6-8]$ but is not recommended when the diagnosis is made on coronary angiography, as it may cause propagation of the dissection and expansion of the intramural hematoma $[2,9,10]$. Treatment-wise, an early report by Thayer et al. [11] recommended bypass grafting for all patients with spontaneous dissection. However, according to DeMaio et al. [1] we agree that, as with any case of severe left main coronary artery disease caused by either atherosclerosis or dissection coronary bypass grafting should be performed. In addition, patients with spontaneous coronary artery dissection who have ongoing ischemia may benefit from revascularisation in attempt to decrease the chance of reinfarction or arrhythmia. In DeMaio's review [1] of all the reported case of follow-up available there was only a $7 \%$ immediate mortality and 3 others $(11 \%)$ died during follow-up at 48,72 , and 132 months. $22(81 \%)$ survived the acute event and all but 1 have remained

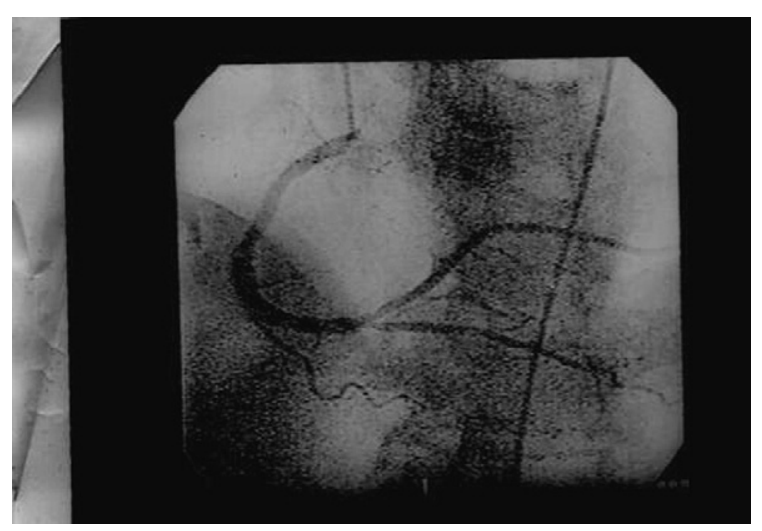

Fig. 4. Angiographic follow-up was performed 5 months later; there was no residual sign. 
asymptomatic. This led the authors to suggest that surgical interventions in these patients be the same as for those with fixed atherosclerotic disease such as left main disease, 3vessel disease or symptoms refractory to medical therapy. After surviving the initial event, all modalities of treatment, including surgery [7], angioplasty with or without stenting [10], cardiac transplant and medical therapy [8], have been employed with a very high success rate. Surgical intervention is indicated in those cases that demonstrate evolving dissections and luminal narrowings resulting in significant hemodynamic compromise. The decision to use angioplasty with or without stenting is operator-dependent and may be done in some cases with long single-vessel dissections or multi-vessel dissections with hemodynamic compromise. Patients with coronary, without any hemodynamic instability or continuing symptoms, should be treated with medical therapy, as most of these patients have shown complete healing of the dissection on follow-up angiography at a later stage including our patient.

We conclude, although surgery was recommended for the all coronary dissection cases, doubtless because of the general evolution presumed, this attitude may no longer be either necessary or wise. We believe that the treatment applied should depend on the clinical signs and symptoms and location and extent of dissection, following the same criteria as for surgical treatment of coronary artery disease. Both fibrinolysis and percutaneous transluminal coronary angioplasty have been successfully used in recent years. Follow-up reports indicate favorable evolution as regards both symptoms and mortality in the survivors of the acute phase and the long-term survival. With the evolution of more invasive methods of treatment it is being recognised more frequently in various clinical settings.

\section{References}

[1] DeMaio SJ, Kinsella SH, Silverman ME. Clinical course and long-term prognosis of spontaneous coronary artery dissection. Am J Cardiol 1999;64:471-4.

[2] Koul AK, Hollander G, Moskovits N, Frankel R, Herrera L, Shani J. Coronary artery dissection during pregnancy and the postpartum period: two case reports and review of literature. Catheter Cardiovasc Interv 2001;52:88-94.

[3] Robinowitz M, Virmani R, McAllister Jr HA. Spontaneous coronary artery dissection and eosinophilic inflammation: a cause effect relationship. Am J Med 1982;72:923-8.

[4] Bukley BH, Roberts WC. Isolated coronary arterial dissection. J Thorac Cardivasc Surg 1978;67:97-102.

[5] Bonnet J, Aumaillet M, Thomas D, Grosgogeat Y, Broustet JP, Bricaud H. Spontaneous coronary artery dissection: case report and evidence fort a defect in collagen metabolism. Eur Heart J 1996;7:904-9.

[6] Boland J, Limet R, Troteur G, Legrand V, Kulbertus H. Left main coronary dissection after mild chest trauma: favorable evolution with fibrinolytic and surgical therapies. Chest 1988;93:213-4.

[7] Pita JP, Gonzalez NV, Alvarez LP, Rodriquez JMV, Beiras AC. Spontaneous coronary artery dissection. Cathet Cardiovasc Diagn 1994;32:27-32.

[8] Montaigne O, Pochmalicki G, Jan F, et al. Primary dissection of the left anterior descending artery. Arch Mal Coeur Vaiss 1992;85:245-8.

[9] Vicary E, Eybel C, Monson D. Survical following spontaneous coronary artery dissection: surgical repair by extrusion of intramural hematoma. Am Heart J 1986;111:593-4.

[10] Klustein MW, Tzivoni D, Bitran D, Mendzelevski B, Ilan M, Almagor Y. Treatment of spontaneous artery dissection: report of three cases. Cathet Cardiovasc Diagn 1997;40:372-6.

[11] Thayer J, Healy R, Maggs P. Spontaneous coronary artery dissection. Ann Thorac Surg 1987;44:97-102. 